\title{
Chennai Water Resource Management Using GIS
}

\author{
Saral Purohit ${ }^{\text {a, } 1}$, Gadhiya Jay Dipakbhai ${ }^{\text {a }}$ and G. Abirami ${ }^{\text {a }}$ \\ ${ }^{a}$ Dept of CSE, SRM Institute of Science and Technology, Tamilnadu, India
}

\begin{abstract}
Water scarcity is one of the biggest problems in the country of India, this problem is often caused by poor water resource management. Notably in India, the city of Chennai has had a water crisis going on for the past few years. On June 19th, 2019, the city officials declared that the city had run out of water. This was termed as 'Day Zero' where there was no water left. Most of the lakes of Chennai dried out. Poor water management and very less rainfall were the major reasons for this scarcity. Water resource management thus is of critical importance to ensure that water is utilized in the right way. So the aim of the proposed system is to evaluate the nature, significance, and rate of change in the water bodies of Chennai over a period of time using GIS/Remote sensing and assess the past and present conditions of water bodies in Chennai and to understand the dynamics and trends of change through various forms of spatial analysis. This is achieved by using different functionalities of ArcGIS and ArcMap and then analysing the obtained data and maps.
\end{abstract}

Keywords. ArcGIS, Water Resource Management, Spatial Analysis, Water Requirement

\section{Introduction}

The water resources in Chennai are mainly provided by Madurantakam Lake and Puzhal lake. The other small lakes near Puzhal such as Kaliveli, Pulicat and Sholavaram these all lakes are located in a $50 \mathrm{~km}$ square area. However, they are now almost dry, according to the Chennai Metropolitan Water Supply and Sewerage Board. Chennai has approximately 40 lakhs ( 0.4 million) wells according to a research in 2012. People living in Chennai have faced a huge scarcity of water due to this crisis. And much of Chennai's water resources are outside the city, it needs better water resource management.

\footnotetext{
${ }^{1}$ Saral Purohit, Dept of CSE, SRM Institute of Science and Technology, Tamilnadu India Email: gd7339@srmist.edu.in.
} 


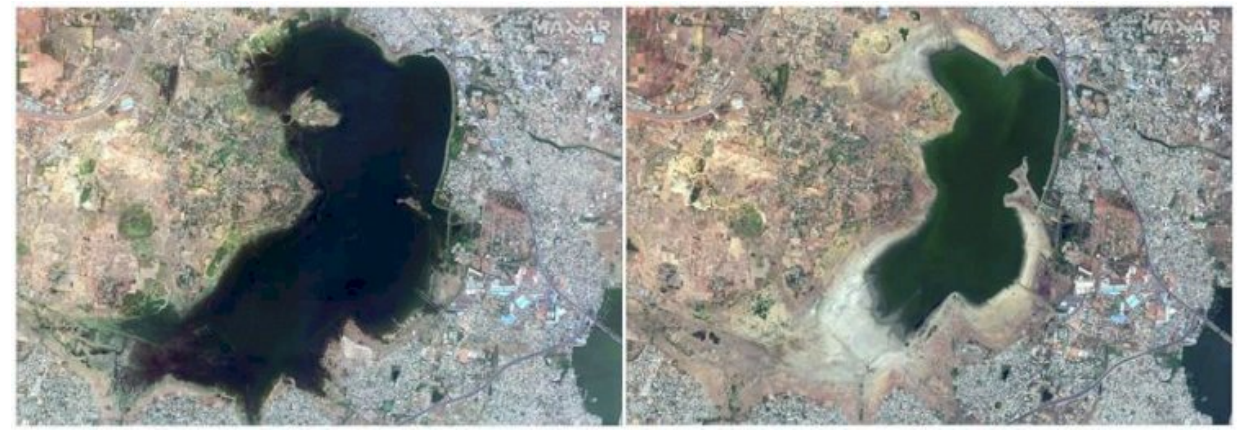

Figure 1.Puzhal lake in Chennai on June 2018 and April 2019

So the aim of the project is to evaluate the nature, significance, and rate of change in the water bodies of Chennai over a period of time using GIS/RS and assess the past and present conditions of water bodies in Chennai and to understand the dynamics and trends of change. Plus create an accurate and up-to-date map of all the water bodies of the city Chennai using GIS Technology with ArcGIS and ArcPy so the tracking of water resources can be much more efficient figure 1.

Geographical Information System is a computer science branch which provides the framework to analyze geographic data. It is also used to display data related positions on the surface of the Earth. The Geographical Information System applies in software and hardware components. GIS applications in water resources include storage of geographic data, hydro-logical management, modeling of groundwater, quality of water, water resource management.

This paper is organized as follows, section 1 is introduction about the research, section 2 is literature review, section 3 is system design, section 4 is implementation and section 5 are conclusion.

\section{Literature Survey}

Cheng Wang [1] provides a real time water resource assessment using WRCC. Tian Yu [2] used the EPANET model which improves calculation efficiency and accuracy and does hydraulic calculation. Da Kong [3] provides a database inquiry for water information and improves the condition of heavy workload with low efficiency.

Rong Li [4] used a three-dimensional display using a GIS platform which provides hydrological analysis. Jiping Liu [5] used a multi water analysis model which helps in water resources allocation. Xuwei $\mathrm{Ru}$ [6] uses a Grid GIS which divides the map in rows and columns.

Tao Sun [7] also provides a 3-dimensional water resource system. The system breaks 2D display and is independent of the GIS platform. Lejiang Guo [8] provides water monitoring and decision analysis for water resources. It also provides social and environmental benefits. Yingzhan $\mathrm{Hu}$ [9] provides flood control decision support, demand analysis and prediction of water resources 
Zhao Mai-huan [10] provides a data analysis function for water resource management which helps in supporting decisions. Qiu Agen [11] provides site selection for water resource management using a real time monitoring system. Majid Akram [12] visualizes the impacts of dewatering on the groundwater. The results show drastic but changes in water resources.

Xianwei Wang [13] uses video image classification and Global Precipitation Measurement for groundwater bodies. Chandan Kumar Singh [14] uses multi criteria analysis for groundwater bodies monitoring areas. A. D. Sheena [15] uses GIS and remote sensing to study the impact of the 2015 Chennai flood. It suggests some water management measures to avoid the impact of floods.

Ashwani Kumar Tiwari [16] uses GIS and water quality index to analyse the groundwater bodies of Ganga and Gomti rivers in India. Kuldeep Tiwari [17] uses an effective and cost saving method for rainwater harvesting in water bodies Rajasthan.

\section{System Design}

\subsection{Study Area Analysis}

Water resource management is one of the most major problems in Chennai. Chennai depends on four main major reservoirs for water which are Red Hills, Chembarambakkam, Cholavaram and Poondi. These reservoirs contribute up to 60 percent of the city's water requirements. But over the past few years, all water bodies in and around the city of Chennai have shrunk and dried up. The average levels of groundwater in the city have also declined rapidly over the fast years. Chennai receives rains in the months of October to January, apart from that there are showers throughout the year.

The city has also been a victim of floods in the year 2015 as well, this explains Chennai's uneven monsoon season over the years. Good water resource management is required in the city, this can be tracked and measured by implementing GIS (Geographical Information System) based models. GIS is excellent for revealing different patterns and dynamic changes that occur in geographical systems over time.

\subsection{Methodology}

The system works on the basis of data management, analysis, capturing, and visualizing data regarding position of points on the surface of Earth. By combining data from various points, GIS helps in understanding spatial data. GIS possesses various powerful tools for analysis this includes analysis of water resources as well. Therefore, a large amount of data can be stored and analyzed using GIS.

\subsection{Architecture}

Considering the need for mapping and tracking water bodies in a city, the water resource management system has a certain set of components needed for its proper functioning, so it's able to map and analyse all data points associated with the water 
bodies of the city of Chennai. Therefore, the system would contain various different components such as -

1. Data Capture and Input Unit

2. GIS Data Files Unit

3. Data Analysis Unit

4. Data Visualization Unit

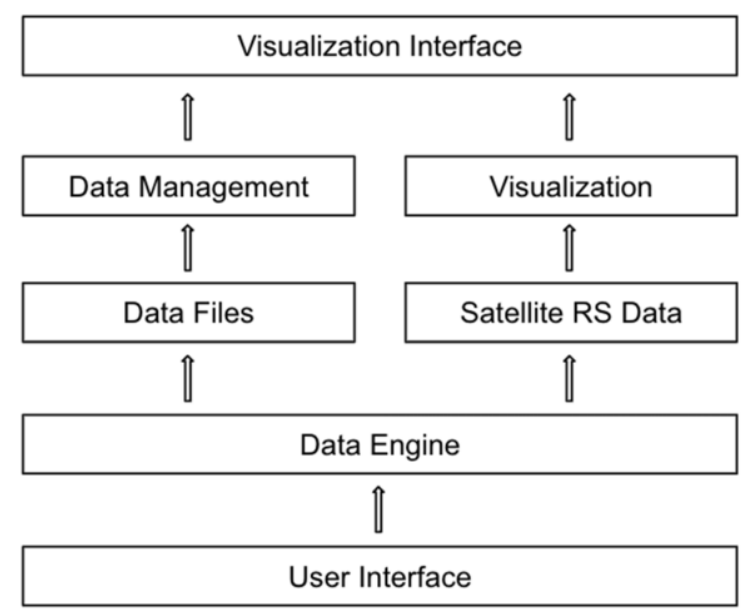

Figure 2. The workflow of the system

In Figure 2. the workflow of the system is shown. Visualization provides analytic tools which helps in 3D modeling and spatial analysis. Data Files include vector data, raster data and images of maps which is used for input analysis. User interface provides users input and output tools to interact with the system.

\section{Implementation}

\subsection{Data Capture and Input}

GIS is used to highlight spatial and geographic relationships among the map objects. The difference between GIS and other mapping systems is that GIS regularly analyze the map and keep the real time mapping updated. The weather features and their relationships all are key analysis of GIS. Thus, GIS is used to map new objects and variables. GIS can also predict the amount of water pollution in river basins. Spatial data include information of roads, mountains, rivers, etc. These data can directly be mapped in ArcGIS. Mainly the data is collected by satellites. Arc GIS allows every data in every format and can be laid on another map. Summing this information together forms Data Capture. Data obtained from satellites in the form of images and objects and converting them to digital format can be provided to GIS technology. Remote sensing is another tool which integrates data with GIS. Remote Sensing includes data from balloons, drones, satellites and planes. 


\subsection{GIS Data Files}

GIS Data files are stored in a database obtained from satellite using software EPANET and ERDAS. By applying ArcView to the map, the map coordinates of the basin are noted. This is the basic data storage in GIS. The major type of data stored is in the format of raster and vector. Raster image represents a grid of pixels and images. They can be viewed in a bitmap display. Commonly used raster images are jpg, gif and png. While vector image is defined on a plan and connected by curves and lines. They are formed based on mathematical equations. And thus, advantageous when zooming in and zooming out plane remains smooth. Commonly used vector images are CDR, AI and SVG.

\subsection{Data Analysis}

GIS makes it easy to perform spatial measurements. Spatial relationship is a distance between two points. These measurements can be done easily by evaluating overlapping areas of two or more maps.

\subsection{Data Visualization}

Visualization is an essential way to visually communicate to others using tools, representation and methods. Visualization can be done in multiple ways, it could be numbers, tables, graphs and charts. For GIS, data visualization is used in spatial and geographical images. These images help in technical analysis of GIS. Many visual tools have been created to support knowledge discovery and data mining.

\subsection{Spatial Analysis}

Spatial Analysis is conducted for the generated map. Where various analysis techniques are utilized. Such as

1. Density Analysis

2. Watershed Analysis

3. Trace Analysis

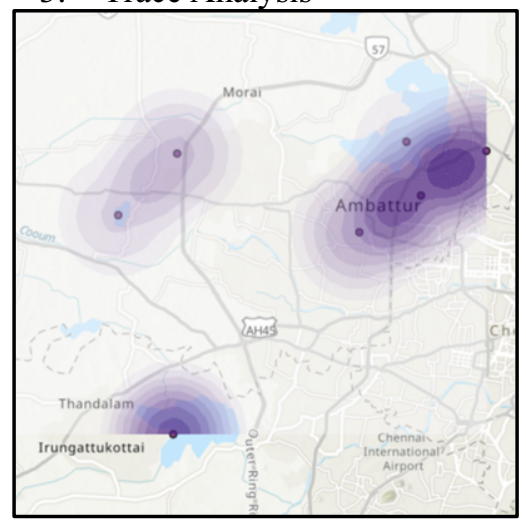

Figure 3. Density Analysis of lakes of Chennai

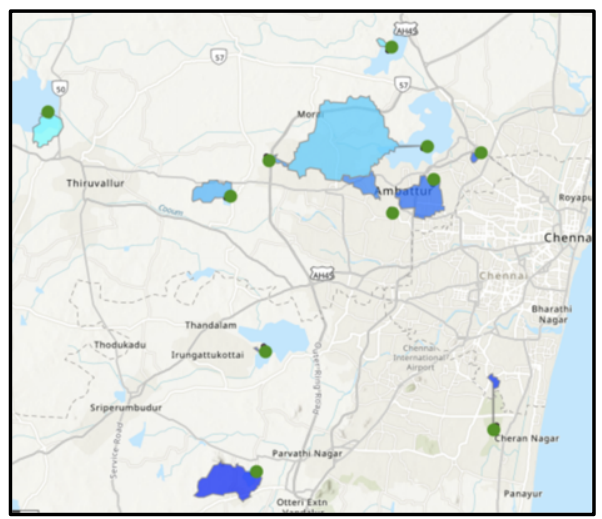

Figure 4.The Watershed analysis of lakes of Chennai 


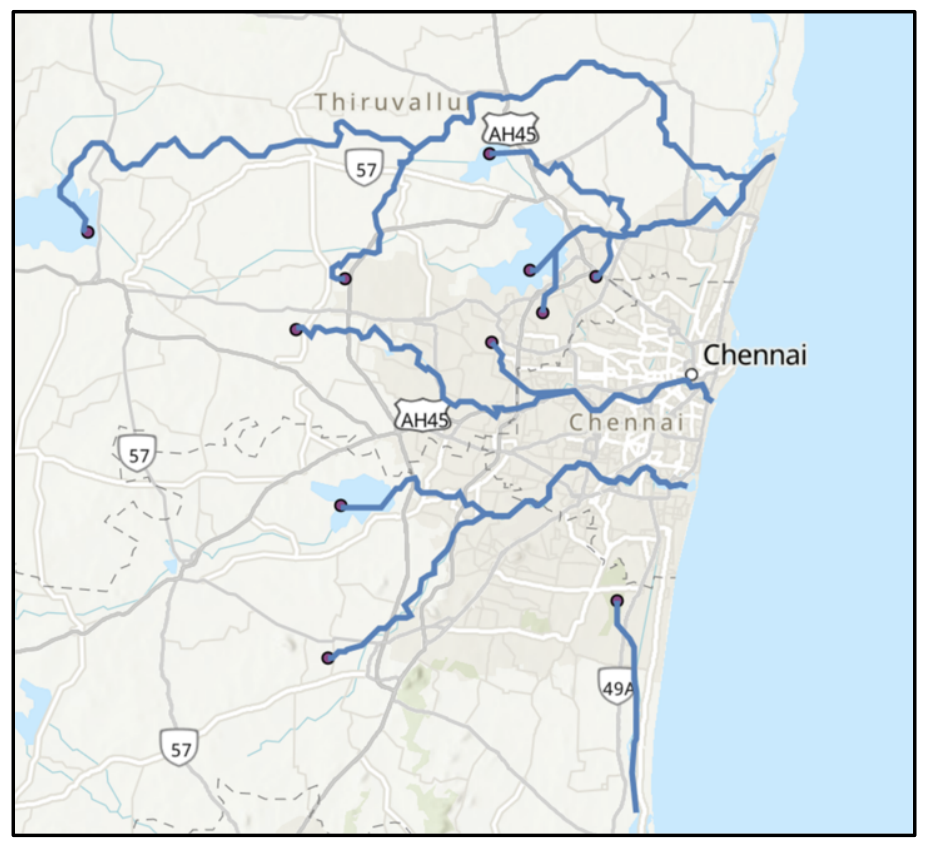

Figure 5. The Downstream analysis of lakes of Chennai

In Figure 3. Density analysis is conducted and it shows concentration and frequency of water bodies' presence across Chennai. In Figure 4. Water analysis is used to create catchment areas based on locations specified for all the water bodies present in Chennai. In Figure 5. Downstream analysis is used to determine the flow paths of all the water bodies in Chennai in a downstream direction.

\section{Conclusion}

Based on the conducted research the idea of implementing a water resource management system using GIS in the city of Chennai. This system can be used for various purposes and projects. The maps and analysis produced by GIS can be quite useful to the government and city officials in getting a broader understanding of Chennai's water resources. It is necessary to continue this research as there are multiple avenues of improvement such as - incorporating different satellite data and using the system for disaster analysis such as flood analysis.

\section{Acknowledgement}

The authors acknowledge and appreciate the support and resources provided by SRM Institute of Science and Technology during the course of the research. 


\section{References}

[1]. Cheng Wang, "Development and Application of GIS-based Analysis System for Studying Water Resources Carrying Capacity in the Shule River Basin", IEEE Paper.

[2]. Tian Yu Ma Liya, "Construction of Water Supply Network Based on GIS and EPANET Model in Fangcun District of Guangzhou0", IEEE Paper

[3]. Da Kong, "Design of water resource management information system based on Web GIS", IEEE Paper.

[4]. Rong Li, Tao Sun, Guo Li, "Application of Three-dimensional GIS to Water Resources", IEEE Paper.

[5]. Jiping Liu, "Research on Optimal Allocation of Water Resources in the Qaidam Basin Based on GIS", IEEE Paper.

[6]. Xuwei Ru, Hongzhi Wu, "Research on Integration and Issuance of Water Resources Information based on Grid GIS", IEEE Paper.

[7]. Rong Li, Tao Sun, Guo Li, "Research on Three-dimensional Water Resources Information System Based on ArcGIS", IEEE Paper.

[8]. Lejiang Guo,Lei Xiao, "Application of GIS and Remote Sensing Techniques for Water Resources Management", IEEE Paper.

[9]. HU Yingzhan, "The Design of Monitoring Management Decision Support System of Water Resources Based on GIS", IEEE Paper.

[10]. Xu Chen-guang, "Geographic Information System Application for Water Allocation", IEEE Paper.

[11]. Qiu Agen, "A Site Selection Algorithm for Water Resources Points Based on GIS Technology", IEEE Paper.

[12]. Elgaali Elgaali; Majid Akram, "Mapping of dewatering impacts on ground water and soil quality using GIS", IEEE Paper.

[13]. Xianwei Wang 1, and Hongjie Xie, "A Review on Applications of Remote Sensing and Geographic Information Systems (GIS) in Water Resources and Flood Risk Management”, MDPI, 2018

[14]. Chandan Kumar Singh, Yashwant B. Katpatal, “A GIS Based Design of Groundwater Level Monitoring Network Using Multi-Criteria Analysis and Geostatistical Method", 2017

[15]. A. D. Sheena, M. Ramalingam, B. Anuradha, "Remote sensing and GIS in the recent Chennai food study", 2018

[16]. Ashwani Kumar Tiwari, Abhay Kumar Singh, Mukesh Kumar Mahato, "Assessment of groundwater quality of Pratapgarh district in India for suitability of drinking purpose using water quality index (WQI) and GIS technique", 2017

[17]. Kuldeep Tiwari, Rohit Goyal, Archana Sarkar, "GIS-based Methodology for Identification of Suitable Locations for Rainwater Harvesting Structures”, 2018 